\title{
Complete genome sequence of Thermosediminibacter oceani type strain (JW/IW-1228 $\mathrm{P}^{\mathrm{T}}$ )
}

\author{
Sam Pitluck ${ }^{1}$, Montri Yasawong ${ }^{2}$, Christine Munk ${ }^{1,3}$, Matt Nolan ${ }^{1}$, Alla Lapidus ${ }^{1}$, Susan \\ Lucas $^{1}$, Tijana Glavina Del Rio ${ }^{1}$, Hope Tice ${ }^{1}$, Jan-Fang Cheng ${ }^{1}$, David Bruce ${ }^{1,3}$, Chris \\ Detter $^{1,3}$, Roxanne Tapia ${ }^{1,3}$, Cliff Han ${ }^{1,3}$, Lynne Goodwin ${ }^{1,3}$, Konstantinos Liolios ${ }^{1}$, Natalia \\ Ivanova ${ }^{1}$, Konstantinos Mavromatis ${ }^{1}$, Natalia Mikhailova ${ }^{1}$, Amrita Pati ${ }^{1}$, Amy Chen ${ }^{4}$, Krishna \\ Palaniappan $^{4}$, Miriam Land ${ }^{1,5}$, Loren Hauser ${ }^{1,5}$, Yun-Juan Chang ${ }^{1,5}$, Cynthia D. Jeffries ${ }^{1,5}$, \\ Manfred Rohde ${ }^{2}$, Stefan Spring ${ }^{6}$, Johannes Sikorski ${ }^{6}$, Markus Göker $^{6}$, Tanja Woyke ${ }^{1}$, James \\ Bristow $^{1}$, Jonathan A. Eisen ${ }^{1,7}$, Victor Markowitz ${ }^{4}$, Philip Hugenholtz ${ }^{1}$, Nikos C. Kyrpides ${ }^{1}$, \\ and Hans-Peter Klenk ${ }^{6 *}$ \\ ${ }^{1}$ DOE Joint Genome Institute, Walnut Creek, California, USA \\ ${ }^{2}$ HZI - Helmholtz Centre for Infection Research, Braunschweig, Germany \\ ${ }^{3}$ Los Alamos National Laboratory, Bioscience Division, Los Alamos, New Mexico, USA \\ ${ }^{4}$ Biological Data Management and Technology Center, Lawrence Berkeley National \\ Laboratory, Berkeley, California, USA \\ ${ }^{5}$ Oak Ridge National Laboratory, Oak Ridge, Tennessee, USA \\ ${ }^{6}$ DSMZ - German Collection of Microorganisms and Cell Cultures GmbH, Braunschweig, \\ Germany \\ ${ }^{7}$ University of California Davis Genome Center, Davis, California, USA \\ *Corresponding author: Hans-Peter Klenk
}

Keywords: chemoorganotroph, anaerobe, thermophile, barophile, upwelling system, core sample, deep sea sediment, Thermoanaerobacterales, Firmicutes, GEBA

\begin{abstract}
Thermosediminibacter oceani (Lee et al. 2006) is the type species of the genus Thermosediminibacter in the family Thermoanaerobacteraceae. The anaerobic, barophilic, chemoorganotrophic thermophile is characterized by straight to curved Gram-negative rods. The strain described in this study was isolated from a core sample of deep sea sediments of the Peruvian high productivity upwelling system. This is the first completed genome sequence of a member of the genus Thermosediminibacter and the seventh genome sequence in the family Thermoanaerobacteraceae. The 2,280,035 bp long genome with its 2,285 protein-coding and 63 RNA genes is a part of the Genomic Encyclopedia of Bacteria and Archaea project.
\end{abstract}

\section{Introduction}

Strain JW/IW-1228P (= DSM 16646 = ATCC BAA1034) is the type strain of Thermosediminibacter oceani, which is the type species of the genus Thermosediminibacter [1], one out of nineteen genera in the family Thermoanaerobacteraceae [24]. The generic name derives from the Greek word 'thermos' meaning 'hot', the Latin word 'sediment' and the Latin word 'bacter' meaning 'a rod or staff', referring to its origin and growth temperature [1]. The species epithet is also derived from the Latin word 'oceani' meaning 'of an ocean', referring to its origin from the ocean [1]. Strain $\mathrm{JW} / \mathrm{IW}-1228 \mathrm{P}^{\mathrm{T}}$ was described in 2005 by Lee et al. as T. oceani [1] and validly published in 2006 [5]. Strain JW/IW-1228P ${ }^{\mathrm{T}}$ was isolated from a core sediment sample (core 201-1228E-1H-1) at 136$143 \mathrm{~cm}$ below the seafloor. The core sample was obtained from the outer shelf edge of the Peruvian high productivity upwelling system. The sea floor there was located at $252 \mathrm{~m}$ below the sea level with $12^{\circ} \mathrm{C}$ mud line temperature. Strain JW/IW$1228 \mathrm{P}^{\mathrm{T}}$ is of particular interest because it is able to ferment a significant number of polysaccharides [1]. Moreover, the strain JW/IW-1228PT is able to use thiosulfate, elemental sulfur and $\mathrm{MnO}_{2}$ as electron acceptors for growth. The only other species in the genus Thermosediminibacter is $T$. litoriperuensis, the type strain of which was isolated from the Peru Trench at 5,086 $\mathrm{m}$ below sea level with a mud-line temperature of $2^{\circ} \mathrm{C}$ [1]. 
Here we present a summary classification and a set of features for $T$. oceani JW/IW-1228P , together with the description of the complete genomic sequencing and annotation.

\section{Classification and features}

The 16S rRNA gene sequence of JW/IW-1228PT is $98.4 \%$ identical to that of $T$. litoriperuensis JW/YJL-1230-2T, the type strain of the only other described species with a validly published name in the genus. The sequence similarities between strain JW/IW-1228P and the type strains of the members of the genera Fervidicola and Caldanaerovirga are $94.4 \%$, with the closest sequence match being that with $F$. ferrireducens and $C$. acetigignens [6]. Three significantly similar $16 \mathrm{~S}$ rRNA gene sequences are known from uncultured clones of Thermovenabulum sp. from GenBank [7]: B5_otu10 (96\%, DQ097675), B14_otu11 (95\%, DQ097676) and B8_otu12 (95\%, DQ097677), all from the Kongdian bed of the Dagang oil field (Hebei province, China) $[7,8]$. No phylotypes from environmental screening or genomic surveys could be linked to the species T. oceani or even the genus Thermosediminibacter, indicating a rather rare occurrence of these in the habitats screened so far (as of July 2010).

Figure 1 shows the phylogenetic neighborhood of T. oceani JW/IW-1228P $\mathrm{P}^{\mathrm{T}}$ in a $16 \mathrm{~S}$ rRNA based tree. The sequences of the three 16S rRNA gene copies in the genome differ from each other by up to one nucleotide and differ by only one nucleotide from the previously published sequence (AY703478).



Figure 1. Phylogenetic tree highlighting the position of $T$. oceani JW/IW-1228 $\mathrm{P}^{\top}$ relative to the type strains of the other species within the family Thermoanaerobacteraceae. The trees were inferred from 1,316 aligned characters $[9,10]$ of the $16 \mathrm{~S}$ rRNA gene sequence under the maximum likelihood criterion [11] and rooted in accordance with the current taxonomy [12]. The branches are scaled in terms of the expected number of substitutions per site. Numbers above branches are support values from 850 bootstrap replicates [13] if larger than 60\%. Lineages with type strain genome sequencing projects registered in GOLD [14] are shown in blue, published genomes in bold [32,33,CP001785,CP001145].

The cells of strain JW/IW-1228 $\mathrm{P}^{\mathrm{T}}$ are straight to curved rods which occur singly, in pairs or in chains (Table 1 and Figure 2). They are between $0.2-0.7 \mu \mathrm{m}$ in diameter and $1.5-16 \mu \mathrm{m}$ in length. In the late-exponential or stationary phase of growth the cells are swollen and subsequently form Lshaped autoplasts [1]. Strain JW/IW-1228PT is Gram-negative, although Thermosediminibacter belongs to the Gram-positive Bacillus-Clostridium subphylum [1]. The cells tend towards elongation and to form aggregates during growth. Motility has not been reported although flagella are observed on the cells (not visible in Figure 2), however, the cells are able to tumble [1], which might imply an impaired flagellar function. Strain JW/IW-1228PT is thermophilic and grows opti- 
mally at $68^{\circ} \mathrm{C}$; the temperature range for growth is $52-76^{\circ} \mathrm{C}$. The optimum $\mathrm{pH}^{25^{\circ} \mathrm{C}}$ for growth is 7.5 , with a range for growth at 6.3-9.3. The optimum salinity for growth is $1 \%(\mathrm{w} / \mathrm{v})$, with a salinity range of $0-6 \%(\mathrm{w} / \mathrm{v})[1]$. Yeast extract is required for growth. The growth of strain JW/IW-1228PT is not observed on $\mathrm{H}_{2} / \mathrm{CO}_{2}(80: 20$, v/v) [1]. The strain produces $\alpha$-glucosidase [22]. The carbon and energy sources used by JW/IW-1228 $\mathrm{P}^{\mathrm{T}}$ include beef extract, casamino acids, cellobiose, fructose, galactose, glucose, inositol, lactate, maltose, mannose, pyruvate, raffinose, sorbitol, sucrose, trehalose, tryptone and xylose when $0.02 \%$ $\mathrm{w} / \mathrm{v}$ of yeast extract is present in growth medium [1]. The fermentation product from glucose is acetate and occasionally trace amounts of propionate, isobutyrate and isovalerate. Acetate is a major product [1]. Strain JW/IW-1228P does not utilize xylitol [22]. It is able to use thiosulfate, elemental sulfur and $\mathrm{MnO}_{2}$ as electron acceptors for growth. There is no indication that JW/IW-1228PT is able to grow chemolithoautotrophically; it does not reduce sulfate or $\mathrm{Fe}(\mathrm{III})$ [1].

\section{Chemotaxonomy}

The peptidoglycan structure of strain JW/IW$1228 \mathrm{P}^{\mathrm{T}}$ is still unknown. The phospholipid fatty acid composition of strain JW/IW-1228PT consists of branched and straight chain saturated acids: iso- $\mathrm{C}_{15: 0}(56.2 \%)$, iso- $\mathrm{C}_{17: 0}(9.6 \%), \mathrm{C}_{16: 0}(7.5 \%)$, anteiso- $\mathrm{C}_{15: 0}(6.7 \%), \mathrm{C}_{16: 1} \omega 9 \mathrm{c}(5.6 \%), \mathrm{C}_{15: 0}(5.0 \%)$, $\mathrm{C}_{18: 1} \omega \mathrm{9c}(3.3 \%)$ and iso- $\mathrm{C}_{16: 0}(1.9 \%)$ [1].

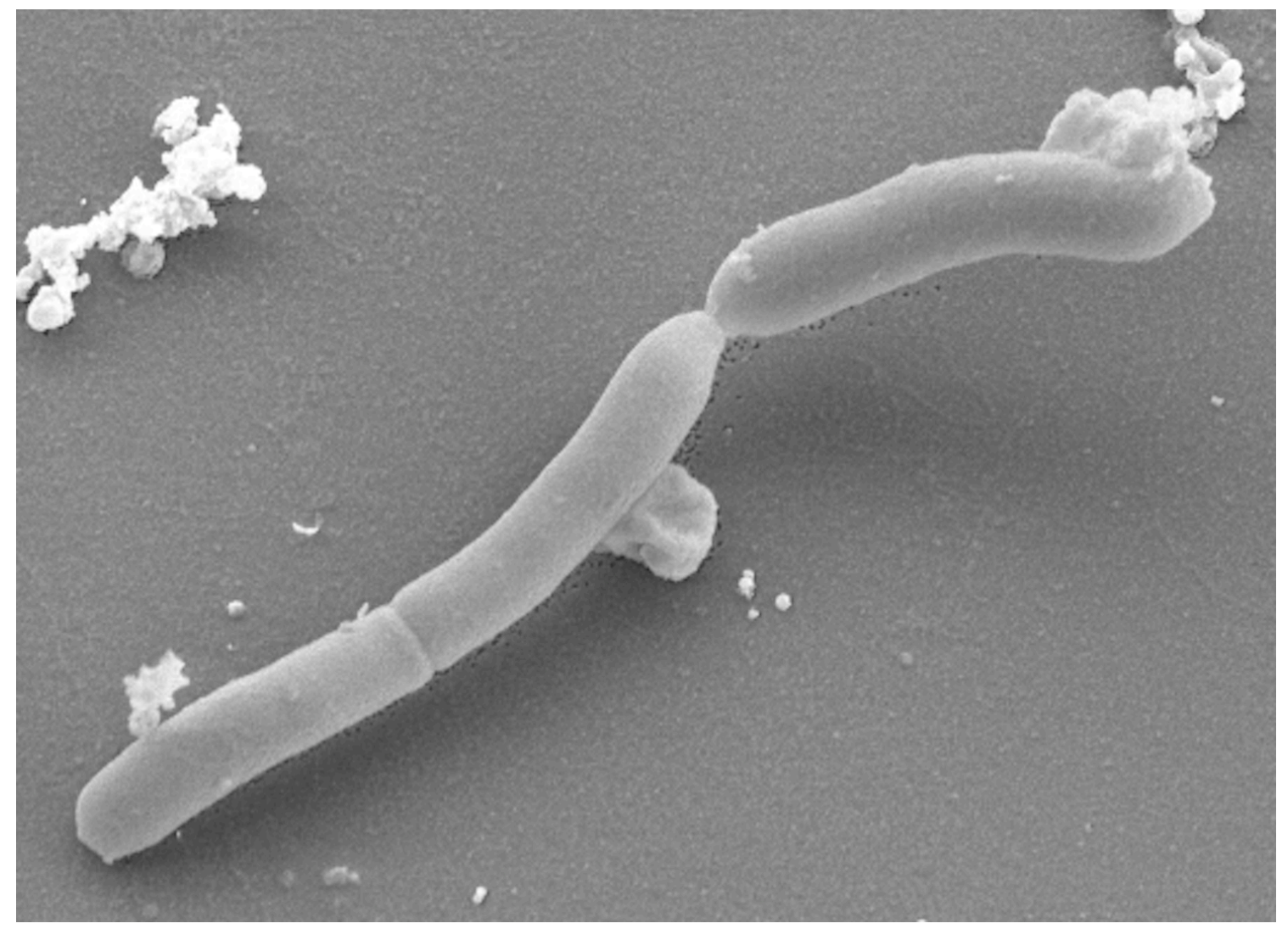

Figure 2. Scanning electron micrograph of T. oceani JW/IW-1228P ${ }^{\mathrm{T}}$

\section{Genome sequencing and annotation Genome project history}

This organism was selected for sequencing on the basis of its phylogenetic position [24], and is part of the Genomic Encyclopedia of Bacteria and Archaea project [25]. The genome project is deposited in the Genome OnLine Database [14] and the complete genome sequence is deposited in GenBank. Sequencing, finishing and annotation were performed by the DOE Joint Genome Institute (JGI). A summary of the project information is shown in Table 2. 
Table 1. Classification and general features of T. oceani JW/IW-1228P according to the MIGS recommendations [15].

\begin{tabular}{|c|c|c|c|}
\hline MIGS ID & Property & 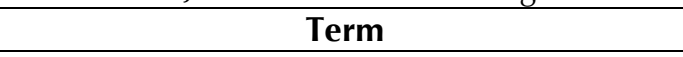 & Evidence code \\
\hline & \multirow{8}{*}{ Current classification } & Domain Bacteria & TAS [16] \\
\hline & & Phylum Firmicutes & TAS $[17,18]$ \\
\hline & & Class Clostridia & TAS $[2,19]$ \\
\hline & & Order Thermoanaerobacterales & TAS $[2,19,20]$ \\
\hline & & Family Thermoanaerobacteraceae & TAS $[2-4]$ \\
\hline & & Genus Thermosediminibacter & TAS $[1,5]$ \\
\hline & & Species Thermosediminibacter oceani & TAS $[1,5]$ \\
\hline & & Type strain JW/IW-1228P & TAS $[1,5]$ \\
\hline & Gram stain & negative & TAS [1] \\
\hline & Cell shape & $\begin{array}{l}\text { straight to curved rods, } 0.2-0.7 \times 1.5-16 \mu \mathrm{m} \text {. } \\
\text { cells tend to elongate and formaggregates. }\end{array}$ & TAS [1] \\
\hline & Motility & no motility, but tumbling (flagella observed) & TAS [1] \\
\hline & Sporulation & not observed & TAS [1] \\
\hline & Temperature range & $52-76^{\circ} \mathrm{C}$ & TAS [1] \\
\hline & Optimum temperature & $68^{\circ} \mathrm{C}$ & TAS [1] \\
\hline & Salinity & $0-6 \% \mathrm{w} / \mathrm{v} \mathrm{NaCl}$ (optimum at $1 \%$ ) & TAS [1] \\
\hline \multirow[t]{3}{*}{ MIGS-22 } & Oxygen requirement & anaerobic & TAS [1] \\
\hline & Carbon source & carbohydrates & TAS [1] \\
\hline & Energy source & chemoorganotroph & TAS [1] \\
\hline MIGS-6 & Habitat & ocean subsurface sediments & TAS [1] \\
\hline MIGS-15 & Biotic relationship & free-living & NAS \\
\hline \multirow[t]{3}{*}{ MIGS-14 } & Pathogenicity & none & NAS \\
\hline & Biosafety level & 1 & TAS [21] \\
\hline & Isolation & core sample from deep sea sediment & TAS [1] \\
\hline MIGS-4 & Geographic location & $\begin{array}{l}\text { subseafloor, outer shelf edge of the Peruvian } \\
\text { high productivity upwelling system, Peru }\end{array}$ & TAS [1] \\
\hline MIGS-5 & Sample collection time & 2002 & NAS \\
\hline MIGS-4.1 & Latitude & 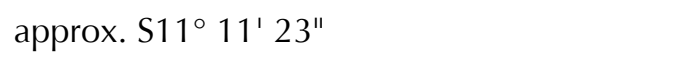 & TAS [22] \\
\hline MIGS-4.2 & Longitude & approx. W79 $4^{\prime} 33^{\prime \prime}$ & TAS [22] \\
\hline MIGS-4.3 & Depth & $136-143 \mathrm{~cm}$ below seafloor & TAS [1] \\
\hline MIGS-4.4 & Altitude & $252 \mathrm{~m}$ below sea level & TAS [1] \\
\hline
\end{tabular}

Evidence codes - IDA: Inferred from Direct Assay (first time in publication); TAS: Traceable Author Statement (i.e., a direct report exists in the literature); NAS: Non-traceable Author Statement (i.e., not directly observed for the living, isolated sample, but based on a generally accepted property for the species, or anecdotal evidence). These evidence codes are from of the Gene Ontology project [23]. If the evidence code is IDA, then the property was directly observed by one of the authors or an expert mentioned in the acknowledgements

\section{Growth conditions and DNA isolation}

T. oceani JW/IW-1228PT, DSM 16646, was grown anaerobically in DSMZ medium 664 (Thermotoga elfii medium) [26] at $68^{\circ} \mathrm{C}$. DNA was isolated from 0.5-1 g of cell paste using Qiagen Genomic 500
DNA Kit (Qiagen, Hilden, Germany) following the standard protocol as recommended by the manufacturer, with modification st/LALMP for cell lysis as described in Wu et al. [25]. 
Table 2. Genome sequencing project information

\begin{tabular}{lll}
\hline MIGS ID & Property & Term \\
\hline MIGS-31 & Finishing quality & Finished \\
MIGS-28 & Libraries used & Tree genomic libraries: one Sanger 8 kb pMCL200 library, one 454 \\
MIGS-29 & Syrosequence standard library and one Illumina standard library \\
MIGS-31.2 & Sequencing platforms & ABI3730, Illumina GAii, 454 GS FLX Titanium \\
MIGS-30 & Assemblers & Newbler version 2.0.0-PostRelease-07/15/2008, Velvet, phrap \\
MIGS-32 & Gene calling method & Prodigal 1.4, GenePRIMP \\
& INSDC ID & CP002131 \\
& Genbank Date of Release & August 5, 2010 \\
& GOLD ID & Gc01361 \\
& NCBI project ID & 30983 \\
& Database: IMG-GEBA & 2503242007 \\
MIGS-13 & Source material identifier & DSM 16646 \\
& Project relevance & Tree of Life, GEBA \\
\hline
\end{tabular}

\section{Genome sequencing and assembly}

The genome was sequenced using a combination of Sanger, Illumina and 454 sequencing platforms. All general aspects of library construction and sequencing can be found at the JGI website. Pyrosequencing reads were assembled using the Newbler assembler version 2.0.0-PostRelease-07/15/2008 (Roche). The initial Newbler assembly consisted of 83 contigs in 32 scaffolds which was converted into a phrap assembly by making fake reads from the consensus. Illumina GAii sequencing data was assembled with Velvet [27] and the consensus sequences were shredded into $1.5 \mathrm{~kb}$ overlapped fake reads and assembled together with the 454 data. Draft assemblies were based on $166.4 \mathrm{Mb}$ 454 draft data and all of the 454 paired end data. Newbler parameters are -consed -a 50 -l 350 -g -m -ml 20. The Phred/Phrap/Consed software package (http://www.phrap.com) was used for sequence assembly and quality assessment in the following finishing process. After the shotgun stage, reads were assembled with parallel phrap (High Performance Software, LLC). Possible misassemblies were corrected with Dupfinisher [28], or sequencing cloned bridging PCR fragments with subcloning. Gaps between contigs were closed by editing in Consed, by PCR and by Bubble PCR primer walks (J.-F.Chang, unpublished). A total of 625 additional reactions and two shatter libraries were necessary to close gaps and to raise the quality of the finished sequence. Illumina data was used to correct potential base errors and increase consensus quality using a software Polisher developed at JGI [29]. The error rate of the com- pleted genome sequence is less than 1 in 100,000 . Together, the combination of the Sanger, Illumina and 454 sequencing platforms provided $65.0 \times$ coverage of the genome.

\section{Genome annotation}

Genes were identified using Prodigal [30] as part of the Oak Ridge National Laboratory genome annotation pipeline, followed by a round of manual curation using the JGI GenePRIMP pipeline [31]. The predicted CDSs were translated and used to search the National Center for Biotechnology Information (NCBI) nonredundant database, UniProt, TIGRFam, Pfam, PRIAM, KEGG, COG, and InterPro databases. Additional gene prediction analysis and functional annotation was performed within the Integrated Microbial Genomes - Expert Review (IMG-ER) platform [32].

\section{Genome properties}

The genome consists of a 2,280,035 bp long chromosome with a $468 \%$ GC content (Table 3 and Figure 3). Of the 2,348 genes predicted, 2,285 were protein-coding genes, and 63 RNAs; eighty eight pseudogenes were also identified. The majority of the protein-coding genes $(73.3 \%)$ were assigned with a putative function while the remaining ones were annotated as hypothetical proteins. The distribution of genes into COGs functional categories is presented in Table 4. 
Table 3. Genome Statistics

\begin{tabular}{lrr}
\hline Attribute & Value & \% of Total \\
\hline Genome size (bp) & $2,280,035$ & $100.00 \%$ \\
DNA coding region (bp) & $1,991,971$ & $87.37 \%$ \\
DNA G+C content (bp) & $1,067,515$ & $46.82 \%$ \\
Number of replicons & 1 & \\
Extrachromosomal elements & 0 & \\
Total genes & 2,348 & $100.00 \%$ \\
RNA genes & 63 & $2.68 \%$ \\
rRNA operons & 3 & \\
Protein-coding genes & 2,285 & $97.32 \%$ \\
Pseudo genes & 88 & $3.75 \%$ \\
Genes with function prediction & 1,722 & $73.34 \%$ \\
Genes in paralog clusters & 366 & $15.59 \%$ \\
Genes assigned to COGs & 1,751 & $74.57 \%$ \\
Genes assigned Pfam domains & 1,925 & $81.98 \%$ \\
Genes with signal peptides & 280 & $11.93 \%$ \\
Genes with transmembrane helices & 563 & $23.98 \%$ \\
CRISPR repeats & 5 & \\
\hline
\end{tabular}

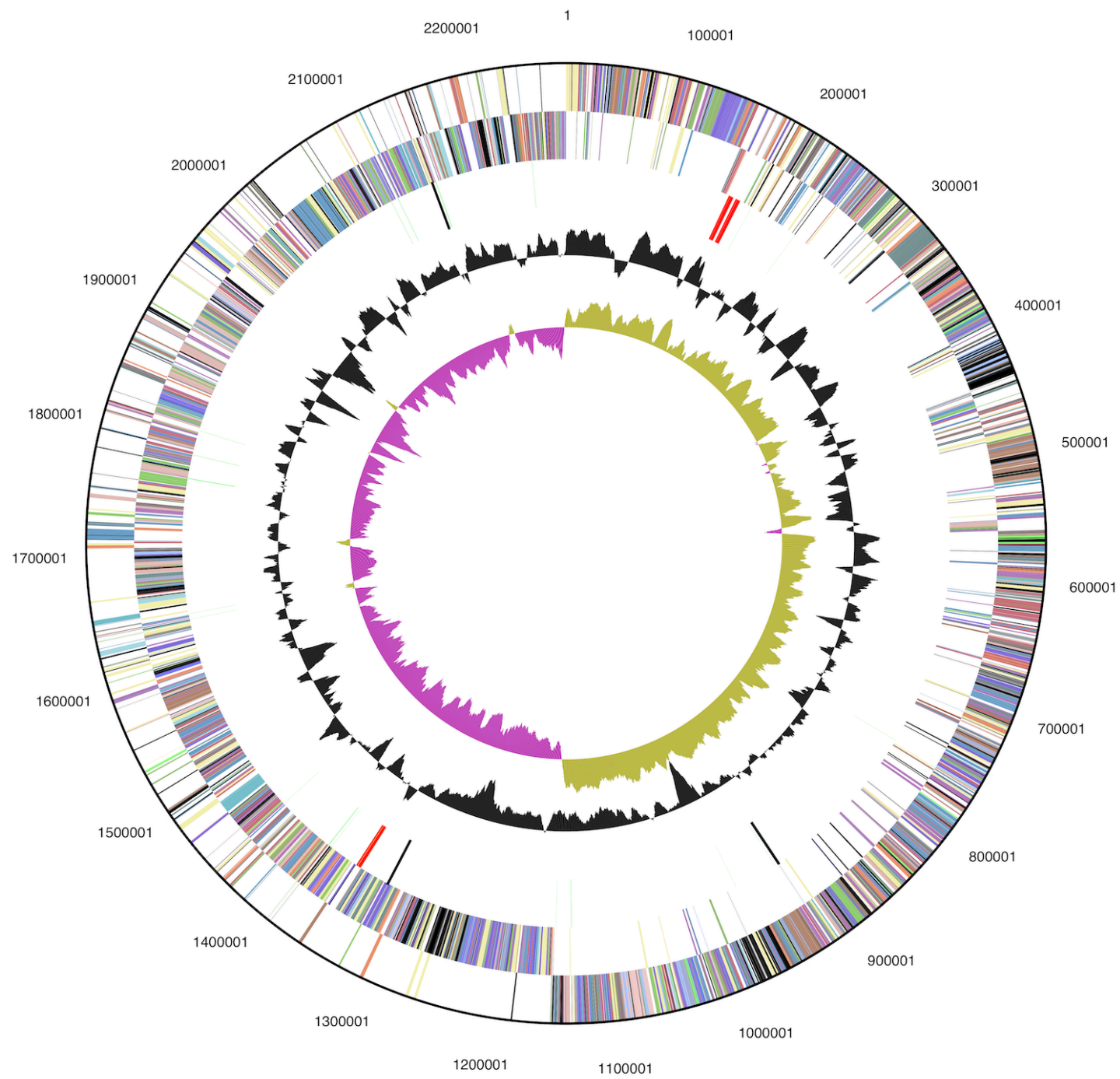

Figure 3. Graphical circular map of the genome. From outside to the center: Genes on forward strand (color by COG categories), Genes on reverse strand (color by COG categories), RNA genes (tRNAs green, rRNAs red, other RNAs black), GC content, GC skew. 
Table 4. Number of genes associated with the general COG functional categories

\begin{tabular}{crrl}
\hline Code & Value & \%age & Description \\
\hline J & 140 & 7.3 & Translation, ribosomal structure and biogenesis \\
A & 0 & 0.0 & RNA processing and modification \\
K & 122 & 6.4 & Transcription \\
L & 191 & 10.0 & Replication, recombination and repair \\
B & 1 & 0.1 & Chromatin structure and dynamics \\
D & 35 & 1.8 & Cell cycle control, cell division, chromosome partitioning \\
Y & 0 & 0.0 & Nuclear structure \\
V & 28 & 1.5 & Defense mechanisms \\
T & 96 & 5.0 & Signal transduction mechanisms \\
M & 107 & 5.6 & Cell wall/membrane/envelope biogenesis \\
N & 58 & 3.0 & Cell motility \\
Z & 0 & 0.0 & Cytoskeleton \\
W & 0 & 0.0 & Extracellular structures \\
U & 49 & 2.6 & Intracellular trafficking and secretion, and vesicular transport \\
O & 58 & 3.0 & Posttranslational modification, protein turnover, chaperones \\
C & 142 & 7.4 & Energy production and conversion \\
G & 117 & 6.1 & Carbohydrate transport and metabolism \\
E & 147 & 7.7 & Amino acid transport and metabolism \\
F & 51 & 2.7 & Nucleotide transport and metabolism \\
H & 94 & 4.9 & Coenzyme transport and metabolism \\
I & 33 & 1.7 & Lipid transport and metabolism \\
P & 85 & 4.5 & Inorganic ion transport and metabolism \\
Q & 25 & 1.3 & Secondary metabolites biosynthesis, transport and catabolism \\
R & 171 & 9.0 & General function prediction only \\
S & 158 & 8.3 & Function unknown \\
- & 597 & 25.4 & Not in COGs \\
\hline & & & \\
& &
\end{tabular}

\section{Acknowledgements}

We would like to gratefully acknowledge the help of Maren Schröder for growing T. oceani cultures and Susanne Schneider for DNA extraction and quality analysis (both at DSMZ). This work was performed under the auspices of the US Department of Energy Office of Science, Biological and Environmental Research Program, and by the University of California, Lawrence Berkeley National Laboratory under contract No. DE-AC02-05CH11231,

\section{References}

1. Lee YJ, Wagner ID, Brice ME, Kevbrin VV, Mills GL, Romanek CS, Wiegel J. Thermosediminibacter oceani gen. nov., sp. nov. and Thermosediminibacter litoriperuensis sp. nov., new anaerobic thermophilic bacteria isolated from Peru Margin.
Lawrence Livermore National Laboratory under Contract No. DE-AC52-07NA27344, and Los Alamos National Laboratory under contract No. DE-AC02-06NA25396, UT-Battelle and Oak Ridge National Laboratory under contract DE-AC05-000R22725, as well as German Research Foundation (DFG) INST 599/1-2 and SI 1352/1-2 and Thailand Research Fund Royal Golden Jubilee Ph.D. Program No. PHD/0019/2548 for MY.
Extremophiles 2005; 9:375-383. PubMed doi:10.1007/s00792-005-0453-4

2. Validation list no. 132. List of new names and new combinations previously effectively, but not validly, published. Int J Syst Evol Microbiol 2010; 60:469-472. doi:10.1099/ijs.0.022855-0 
3. Wiegel J. 2009. Family I. Thermoanaerobacteraceae fam. nov., p. 1225. In P De Vos, GM Garrity, D.Jones, NR Krieg, W Ludwig, FA Rainey, KH Schleifer, and W Whitman (eds), Bergey's Manual of Systematic Bacteriology, second ed, vol. 3. Springer-Verlag, New York.

4. Garrity G. NamesforLife. BrowserTool takes expertise out of the database and puts it right in the browser. Microbiol Today 2010; 7:1.

5. Validation list no. 109. List of new names and new combinations previously effectively, but not validly, published. Int I Syst Evol Microbiol 2006; 56:925-927. PubMed doi:10.1099/ijs.0.64380-0

6. Chun J, Lee JH, Jung $Y, \operatorname{Kim} M, \operatorname{Kim} S$, Kim BK, Lim YW. EzTaxon: a web-based tool for the identification of prokaryotes based on $16 \mathrm{~S}$ ribosomal RNA gene sequences. Int / Syst Evol Microbiol 2007; 57:2259-2261. PubMed doi:10.1099/ijs.0.64915-0

7. Benson DA, Karsch-Mizrachi I, Lipman DJ, Ostell J, Sayers EW. GenBank. Nucleic Acids Res 2009; 37:D26-D31. PubMed doi:10.1093/nar/gkn723

8. Nazina TN, Shestakova NM, Grigor'ian AA, Mikhailova EM, Turova TP, Poltaraus AB, Feng C, Ni F, Beliaev SS. Phylogenetic diversity and activity of anaerobic microorganisms of high-temperature horizons of the Dagang Oilfield (China). Mikrobiologiia 2006; 75:70-81. PubMed

9. Castresana J. Selection of conserved blocks from multiple alignments for their use in phylogenetic analysis. Mol Biol Evol 2000; 17:540-552. PubMed

10. Lee C, Grasso C, Sharlow MF. Multiple sequence alignment using partial order graphs. BioinformatiCS 2002; 18:452-464. PubMed doi:10.1093/bioinformatics/18.3.452

11. Stamatakis A, Hoover P, Rougemont J. A rapid bootstrap algorithm for the RAxML Web servers. Syst Biol 2008; 57:758-771. PubMed doi:10.1080/10635150802429642

12. Yarza P, Richter M, Peplies J, Euzeby J, Amann R, Schleifer KH, Ludwig W, Glöckner FO, RossellóMóra R. The All-Species Living Tree project: A $16 \mathrm{~S}$ rRNA-based phylogenetic tree of all sequenced type strains. Syst Appl Microbiol 2008; 31:241-250. PubMed doi:10.1016/j.syapm.2008.07.001

13. Pattengale ND, Alipour $M$, Bininda-Emonds ORP, Moret BME, Stamatakis A. How many bootstrap replicates are necessary? Lect Notes Comput Sci
2009; 5541:184-200. doi:10.1007/978-3-64202008-7_13

14. Liolios K, Chen IM, Mavromatis K, Tavernarakis $\mathrm{N}$, Hugenholtz P, Markowitz VM, Kyrpides NC. The Genomes On Line Database (GOLD) in 2009: status of genomic and metagenomic projects and their associated metadata. Nucleic Acids Res 2010; 38:D346-D354. PubMed doi:10.1093/nar/gkp848

15. Field D, Garrity G, Gray T, Morrison N, Selengut J, Sterk P, Tatusova T, Thomson N, Allen MJ, Angiuoli SV, et al. The minimum information about a genome sequence (MIGS) specification. Nat Biotechnol 2008; 26:541-547. PubMed doi:10.1038/nbt1360

16. Woese CR, Kandler O, Wheelis ML. Towards a natural system of organisms: proposal for the domains Archaea, Bacteria, and Eucarya. Proc Natl Acad Sci USA 1990; 87:4576-4579. PubMed doi:10.1073/pnas.87.12.4576

17. Garrity GM, Holt JG. 2001. The Road Map to the Manual, p. 119-169. In G. M. Garrity, D. R. Boone, and R. W. Castenholz (ed.), Bergey's Manual of Systematic Bacteriology, 2 ed, vol. 1. Springer, New York.

18. Gibbons NE, Murrey RGE. Proposals concerning the higher taxa of bacteria. Int I Syst Bacteriol 1978; 28:1-6. doi:10.1099/00207713-28-1-1

19. Rainey FA. 2009. Class II. Clostridia class nov, p. 736. In: P De Vos, GM Garrity, D Jones, NR Krieg, W Ludwig, FA Rainey, KH Schleifer, W Whitman (eds), Bergey's Manual of Systematic Bacteriology, second edition, vol. 3 (The Firmicutes), vol. 3. Springer, Dordrecht, Heidelberg, London, New York.

20. Wiegel J. Order III. Thermoanaerobacterales ord. nov. In: De Vos P, Garrity G, Jones D, Krieg NR, Ludwig W, Rainey FA, Schleifer KH, Whitman WB (eds), Bergey's Manual of Systematic Bacteriology, Second Edition, Volume 3, SpringerVerlag, New York, 2009, p. 1224.

21. Classification of bacteria and archaea in risk groups. TRBA 466.

22. D'Hondt SL, Jørgensen BB, Miller DJ, Aiello IW, Bekins B, Blake R, Cragg BR, Cypionka H, Dickens GR, Ferdelman T, et al. Init Repts, 201: College Station, TX (Ocean Drilling Program) 2003.

23. Ashburner M, Ball CA, Blake JA, Botstein D, Butler H, Cherry JM, Davis AP, Dolinski K, Dwight SS, Eppig JT, et al. Gene Ontology: tool for the 
unification of biology. Nat Genet 2000; 25:25-29. $\underline{\text { PubMed doi: } 10.1038 / 75556}$

24. Klenk HP, Göker M. En route to a genome-based classification of Archaea and Bacteria? Syst Appl Microbiol 2010; 33:175-182. PubMed doi:10.1016/j.syapm.2010.03.003

25. Wu D, Hugenholtz P, Mavromatis K, Pukall R, Dalin E, Ivanova NN, Kunin V, Goodwin L, Wu $M$, Tindall BJ, et al. A phylogeny-driven genomic encyclopaedia of Bacteria and Archaea. Nature 2009; 462:1056-1060. PubMed $\underline{\text { doi:10.1038/nature08656 }}$

26. List of growth media used at DSMZ: http://www.dsmz.de/microorganisms/media_list.p hp.

27. Zerbino DR, Birney E. Velvet: algorithms for de novo short read assembly using de Bruijn graphs. Genome Res 2008; 18:821-829. PubMed doi:10.1101/gr.074492.107

28. Han C, Chain P. Finishing repeat regions automatically with Dupfinisher. In: Proceeding of the 2006 international conference on bioinformatics
\& computational biology. HR Arabnia \& H Valafar (eds), CSREA Press. June 26-29, 2006; 141 146.

29. Lapidus A, LaButti K, Foster B, Lowry S, Trong S, Goltsman E. POLISHER: An effective tool for using ultra short reads in microbial genome assembly and finishing. AGBT, Marco Island, FL, 2008.

30. Hyatt D, Chen GL, Locascio PF, Land ML, Larimer FW, Hauser LJ. Prodigal: prokaryotic gene recognition and translation initiation site identification. BMC Bioinformatics 2010; 11:119. $\underline{\text { PubMed doi:10.1186/1471-2105-11-119 }}$

31. Pati A, Ivanova NN, Mikhailova N, Ovchinnikova G, Hooper SD, Lykidis A, Kyrpides NC. GenePRIMP: a gene prediction improvement pipeline for prokaryotic genomes. Nat Methods 2010; 7:455-457. PubMed doi:10.1038/nmeth.1457

32. Markowitz VM, Ivanova NN, Chen IMA, Chu K, Kyrpides NC. IMG ER: a system for microbial genome annotation expert review and curation. Bioinformatics 2009; 25:2271-2278. PubMed doi:10.1093/bioinformatics/btp393 